\title{
Authors' reply to comments on Heidari et al.: Association between serum vitamin $D$ level and gonarthrosis
}

\author{
Behzad Heidari • Parham Heidari • Karim Hajian
}

Received: 19 February 2011 / Accepted: 19 February 2011/Published online: 10 March 2011

(C) Springer-Verlag 2011

\section{Dear Editor,}

We thank Drs. Gursu and Yıldırım for their interest in our article on the association of vitamin D deficiency and knee osteoarthritis [1]. They addressed the question of whether low serum 25-hydroxyvitamin D (25-OHD) in patients with knee OA may be the result of knee osteoarthritis. They argued that these patients may have a sedentary life and inactivity due to knee pain which leads to less sunlight exposure and consequent lower serum 25-OHD level compared with the general population.

Although this explanation may be applicable in patients with severe limitation of physical activities, in our study patients with limitation of physical activity were not included and therefore the low serum 25-OHD levels can not be attributed to knee pain and it's consequences.

It is important to remember that in cross-sectional studies the true direction of causality is unclear and can be elucidated only in longitudinal studies.

The results of our study provided information that vitamin $\mathrm{D}$ deficiency is not the result of knee OA but an associated factor of knee OA. This is because in knee OA factors other than pain such as degree of psychological involvement, quadriceps strength, body weight and age are also significantly correlated with disability [2-4]. In elderly subjects quadriceps

B. Heidari $(\bowtie)$

Department of Internal Medicine, Division of Rheumatology,

Babol University of Medical Sciences,

Babol, Persia, Iran

e-mail: heidaribeh@yahoo.com

P. Heidari

Faculty of Veterinary Medicine, Azad Islamic University,

Tehran, Iran

K. Hajian

Department of Social Medicine, Babol University of Medical

Sciences,

Babol, Persia, Iran strength, knee pain and age were the most important determinants of functional impairment [3]. With increasing age, disability due to knee pain would also increase [3]. Therefore, if serum 25-OHD is being considered as a result of knee pain and it's subsequent functional disability, then older subjects with knee OA might have lower serum 25-OHD level compared with younger patients; whereas in contrast, serum 25-OHD levels in patients $>60$ years was higher than in those younger than 60 years, and was particularly greater than in patients aged less than 55 years. Hence, low 25-OHD in this study can not be attributed to knee OA.

The observed association in this study does not represent causality, and serum 25-OHD deficiency in this study has not been considered as an aetiological factor of knee OA. However, presence of a dose-response relationship [see Table 2 in 5] may indicate a contributive role for vitamin D deficiency in the development of knee OA, and it suggests a placebo-controlled prospective study with intervention to evaluate the effect of vitamin D repletion on knee pain in patients with knee OA. Response to vitamin D supplement provides further data in this regard. However, further studies are needed to elucidate the contributive role of vitamin D in the development or progression of osteoarthritis.

\section{References}

1. Heidari B, Heidari P, Hajian K (2011) Association between serum vitamin $\mathrm{D}$ deficiency and knee osteoarthritis. Int Orthop [Epub ahead of print]

2. Salaffi F, Cavalieri F, Nolli M, Ferraccioli G (1991) Analysis of disability in knee osteoarthritis. Relationship with age and psychological variables but not with radiological score. J Rheumatol 18:1581-1586

3. McAlindon TE, Cooper C, Kirwan JR, Dieppe PA (1993) Determinants of disability in osteoarthritis of the knee. Ann Rheum Dis 52:258-262

4. McAlindon TE, Cooper C, Kiwan JR, Dieppe PA (1992) Knee pain and disability in the community. Br J Rheumatol 31:189-192

5. Wright RJ (2005) Make no bones about it. Editorial. Chest 128:3781-3783 